19 Revue d'histoire du XIXe siècle

Société d'histoire de la révolution de 1848 et des

révolutions du XIXe siècle

$39 \mid 2009$

Le monde de l'imprimé: des territoires aux acteurs -

Education et politique - Histoires politiques

\title{
Les cours secondaires pour jeunes filles à Troyes sous le Second Empire, entre autorités municipales et administration bonapartiste
}

The secondary classes for young women in Troyes during the Second Empire, between local authorities and the Bonapartist government

\section{Yves Verneuil}

\section{OpenEdition \\ Journals}

Édition électronique

URL : http://journals.openedition.org/rh19/3924

DOI : $10.4000 /$ rh 19.3924

ISSN : $1777-5329$

Éditeur

La Société de 1848

Édition imprimée

Date de publication : 10 décembre 2009

Pagination : 95-111

ISSN : 1265-1354

Référence électronique

Yves Verneuil, «Les cours secondaires pour jeunes filles à Troyes sous le Second Empire, entre autorités municipales et administration bonapartiste », Revue d'histoire du XIXe siècle [En ligne] 39 | 2009, mis en ligne le 15 décembre 2012, consulté le 30 avril 2019. URL : http:// journals.openedition.org/rh19/3924; DOI : 10.4000/rh19.3924 


\section{DENYS BARAU}

\section{La dimension éducative du mouvement philhellène!}

Le soulèvement des Grecs contre la domination turque en 1821 a suscité un mouvement en leur faveur qui, par vagues successives et jusqu'en 1829, a touché la plupart des pays d'Europe. Ce mouvement s'est manifesté sous des formes très diverses - depuis l'organisation de souscriptions ou de concerts ou la publication de poèmes jusqu'au départ de volontaires pour combattre aux côtés des insurgés - souvent sous l'impulsion de comités formés spécialement : les "comités grecs $»^{2}$. À leurs débuts, en 1825, ceux de Paris et de Genève ont imaginé comme un moyen d'action de prendre en charge l'éducation de quelques jeunes garçons $s^{3}$. En se séparant, en 1830, le comité de Genève et celui de Berlin ont voulu consacrer à des actions du même genre leurs fonds restants ${ }^{4}$. Entre temps, des sociétés spécialisées s'étaient formées en Angleterre et en Suisse. Pareille insistance ne peut qu'être symptomatique : pour en découvrir les raisons, nous en explorerons d'abord les manifestations; nous en rechercherons ensuite les échos dans d'autres aspects du philhellénisme.

1. Ce texte reprend une communication à un colloque organisé à Corfou par l'Université Ionienne (3-5 mai 2007); une traduction grecque figure dans les actes de ce colloque (H E $\lambda \lambda \eta v i ́ x \eta ́ ~ E \pi \alpha v \alpha \sigma \tau \alpha \sigma \eta$

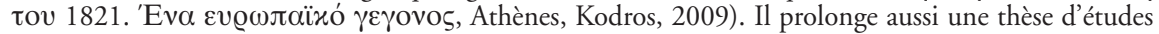
politiques, La cause des Grecs. Essai historique sur le mouvement philhellène (1821-1829), sous la direction de Pierre Rosanvallon, EHESS, 2006, et dont une version remaniée a été publiée sous le titre La cause des Grecs. Une histoire du mouvement philhellène (1821-1829), Paris, Honoré Champion, 2009.

2. Il y en a eu trois générations : la première active en Allemagne rhénane et en Suisse en 1821 et 1822; la seconde en 1823 et 1824 surtout anglaise et américaine. Ceux dont il est question ici sont les principaux d'une troisième vague, à l'apogée du mouvement en 1825 et 1826, étendue à presque toute l'Europe continentale. Formés de gens en vue, et, pour Paris, très liés au monde des salons, politiquement pluralistes (celui de Paris rassemblait les principaux chefs libéraux - Casimir Périer, Laffitte, Sébastiani ou Ternaux -, Chateaubriand et le duc de Fitz-James, intime de Charles X), ils ont été les centres d'initiative d'un mouvement qui a affecté en particulier les milieux des gens de loi (avocats, notaires), du commerce, de l'industrie et de la finance, et aussi le monde militaire, les femmes, la jeunesse. On pourra consulter William Saint Clair, That Greece Might Still be Free. The Philhellens in the War of Independence, Oxford, Oxford University Press, 1972 et mon livre.

3. Pour Paris, lettre de J.-G. Eynard, 5 février 1825, Bibliothèque de Genève (Bib. Genève), Ms. sup 1871. Pour Genève, séance du 15 octobre 1825 (Bib. Genève, Ms. 491).

4. Comité de Genève, séance du 18 mars 1830 (Ms. 491) et lettre du comité de Berlin à J.-G. Eynard, 3 octobre 1829 (Bib. Genève, Ms. sup 1886, fo 314 ). 


\section{DES INITIATIVES DIVERSES DANS LEURS INSPIRATIONS ET DANS LEURS MODALITÉS}

Soulignons d'abord le rôle ici du calcul politique ou des élans de la compassion. Certains des enfants étaient orphelins, ou leurs familles avaient été éprouvées par la guerre : les prendre en charge était une façon parmi d'autres d'aider les victimes. Par exemple, un réseau de comités français, suisses et allemands se mobilisait, en 1826 et 1827, pour offrir aux deux fils d'un négociant de Chio l'éducation dont les avait privés le meurtre de leur père lors du massacre d'avril 1822 : on apportait aussi par là une consolation à toute la famille ${ }^{5}$. Et la question de l'éducation des enfants s'est posée à plus grande échelle quand on a voulu racheter les Grecs réduits en esclavage après la chute de Missolonghi : parmi eux, il y avait beaucoup d'enfants, sans famille souvent. Une institution aux environs de Bâle leur était réservée.

Dans cet esprit, Charles $\mathrm{X}$ avait voulu faire venir en France une cinquantaine d'enfants rachetés en Égypte. Arrivés à Toulon, on les renvoya pourtant aussitôt en Morée, en alléguant le coût excessif, les risques ( «es maladies et de funestes habitudes $\left.{ }^{6}\right)$. Le récent changement de ministère avait sans doute pesé davantage. Générosité personnelle du roi, le projet était aussi un geste politique, en harmonie avec l'expédition de Morée, décidée par le ministère Martignac. Celui de Polignac, sans changer de ligne, y mettait moins d'enthousiasme. N'eût-il pas été politique dans son inspiration, ce revirement le devint par ses effets : révélée par la presse, l'affaire offrait un argument à l'opposition. Autant que l'avenir de ces enfants, les relations de la France avec le nouvel État étaient en jeu. Comme déjà dans le projet du comité de Paris où l'on imaginait ces enfants ramenant plus tard dans leur patrie «l'amour de la France et le désir de voir se former des liens fraternels entre les deux États $»^{7}$. Un des initiateurs du projet, le financier genevois Jean-Gabriel Eynard, en donnait une autre interprétation non moins politique : en se chargeant de l'éducation des fils de chefs militaires, le comité se donnerait un moyen d' "exciter le courage et le zèle des Grecs» et pourrait "avoir sur [ces chefs] une influence morale utile $»^{8}$. La présence de ces enfants de héros prestigieux a servi aussi à entretenir la mobilisation de l'opinion. Nicolas Canaris devait être une des attractions du concert qui, fin avril 1826, marqua

5. Bib. Genève, Ms. 3228, fo 99-102, 160-165; Ms. sup. 491, séances des 14 juillet et 15 août 1826, 13 janvier, 12 février et 3 mars 1827; Journal de la Société de la Morale chrétienne, n 44, p. 113118.

6. Archives nationales (Arch. nat.), F7 6722, dossier 43, et Bib. Genève, Ms. sup. 1871, note de J.-G. Eynard du 30 octobre 1829. Dans les instructions aux inspecteurs de l'Instruction publique en 1822 , il est dit que «l'air de santé et de contentement qui règnent sur le visage des élèves sont d'ordinaire [...] une marque qu'ils n'ont point d'habitudes funestes" (cité par Maurice Gontard, L'enseignement secondaire en France de la fin de l'Ancien Régime à la loi Falloux, 1750-1850, Aix-en-Provence, Édisud, 1984 , p. 127); la formule rappelle aussi les «funestes secrets» désignant les pratiques contraceptives, et devait viser ici les mœurs prêtées aux Ottomans (onanisme, homosexualité).

7. Le Constitutionnel, 21 février 1825.

8. Bib. Genève, Ms. sup. 1871, lettres de J.-G. Eynard, 9 et 13 février 1825. 
à Paris le point d'orgue de la campagne pour Missolonghi. Poussant plus loin l'utilisation spectaculaire et propagandiste, en septembre 1824, Edward Blaquière, du comité de Londres, avait fait parader habillés en costume national, dans les rues de la ville deux des enfants qu'il ramenait pour être éduqués à l'école de Lambeth'.

Voir «mettre en spectacle les rejetons des héros de la Grèce moderne" indignait un ancien collaborateur de Pestalozzi, Emmanuel de Fellenberg, qui avait créé sur son domaine de Hofwil, près de Berne, une école expérimentale ${ }^{10}$. Un autre pédagogue, le Genevois Adolphe Peschier-Vieusseux, prenait ses distances par rapport au ressort compassionnel en prévoyant, pour un projet destiné à des jeunes filles, de «choisir non pas les plus pauvres, mais les plus intelligentes $»^{11}$. Chacun à sa manière, ils entendaient donner à ces initiatives toute leur portée éducative. À leur suite, voyons à présent comment des pratiques et des objectifs proprement pédagogiques se dégageaient des intentions charitables ou politiques.

La pédagogie a pesé bien moins dans la sélection de cette petite population que les malheurs de leurs familles, ou le nom de leurs pères: Paris a eu le fils de Canaris, Genève, celui de l'amiral Tombazis, Munich, un Miaoulis et un Botzaris... Quant à leur très petit nombre (une vingtaine en Angleterre, 19 à Paris ${ }^{12}$, pas plus de trois à la fois à Lausanne $e^{13}$, au mieux 18 à l'orphelinat de Bâle $\left.{ }^{14}\right)$, il tenait d'abord à des moyens limités. Seuls Fellenberg et Peschier avançaient des critères pédagogiques. Peschier ne s'inquiétait pas que de l'intelligence des candidates : observant que, dans une famille à laquelle on s'intéressait à Genève, la fille cadette de dix ans, était seule susceptible d'éducation, il faisait de l'âge un critère pédagogique ${ }^{15}$. Fellenberg, qui refusait aussi de s'occuper de "jeunes Grecs déjà avancés en âge " ", n'entendait, lui, recevoir que six enfants, et choisis parmi les montagnards du Péloponnèse, "descendants de l'ancienne Sparte», les seuls à offrir le "fond de solidité et de détermination" nécessaire ${ }^{17}$.

Souvent très jeunes, ces enfants, s'ils n'étaient pas orphelins, étaient séparés des leurs pour longtemps (huit ans pour Nicolas Canaris ${ }^{18}$ ); il fallait donc aussi une prise en charge familiale. Constantin Canaris et Emmanuel

9. William Saint Clair, That Greece Might Still be Free..., ouv. cité, p. 213-214. Il cite un article du New Monthly Magazine, ${ }^{\circ} 12,1824$.

10. Bib. Genève, Ms. sup. 1885, fo $252-253$.

11. Bib. Genève, Ms. sup. 1886, fo 134-135.

12. D'après les comptes publiés dans la revue Documents relatifs à l'état présent de la Grèce.

13. Bib. Genève, Ms. 3228, fo 158-159 et Ms. sup. 1891, fo 156-157.

14. Bib. Genève, Ms. 3228, fo 18-19.

15. Bib. Genève, Ms. sup. 1886, f $\mathrm{f}^{\circ}$ 165-166.

16. Bib. Genève, Ms. sup. 1885 , fo $252-253$.

17. Bib. Genève, Ms. sup. 1885, fo 221.

18. Il le rappelle, sans en dire plus, dans une lettre de mai 1839, où il raconte l'échec de sa tentative pour être admis à Polytechnique, un espoir qui aurait sans doute moins bien résisté aux avertissements de son père, si le souvenir de son premier séjour n’avait été heureux, Bib. Genève, Ms. sup. 1886, fo $185-186$. 
Tombazis promettaient à leurs fils de «nouveaux pères» (le comité de Paris) ou un "second père" (Jean-Gabriel Eynard) ${ }^{19}$. Il y eut même de véritables adoptions comme celle d'un neveu de Canaris, par l'architecte Mouchonnet $^{20}$. Mais les deux registres, familial et scolaire, ne se distinguaient pas toujours bien : un des pupilles de la Société philhellénique du Canton de Vaud qui venait de mourir avait été traité, disait-on, par l'instituteur pendant sa maladie "avec l'affection qu'on porte à un jeune frère $»^{21}$. Les membres de la Société se montraient du reste plus préoccupés de la santé de leurs protégés que du progrès de leurs études, dont on se félicitait de façon plutôt mécanique.

Cet instituteur était-il un maitre de pension chez qui les enfants étaient placés ou une sorte de précepteur spécialement embauché? On ne sait, mais on aperçoit là deux voies possibles pour assurer la partie scolaire du projet. D'ordinaire les enfants ont été confiés à des établissements déjà existants : en Angleterre, l'école centrale lancastérienne de Lambeth ${ }^{22}$; à Paris, surtout le Collège Sainte-Barbe ${ }^{23}$; à Genève, la pension de Rodolphe Töpffer. Pour le choix, on a considéré et les sympathies des maitres pour la cause ${ }^{24}$ et les qualités professionnelles. Ce qui n'excluait pas toujours un certain amateurisme : Peschier pensait accueillir ses jeunes Grecques dans ses cours de littérature et confier leur éducation au zèle bénévole de dames philhellènes. Ce n'étaient que des filles, il est vrai... Pour les garçons, ils ont reçu, semble-t-il, à Lambeth ou à Sainte-Barbe le même enseignement que leurs condisciples français ou anglais ${ }^{25}$. En revanche, Emmanuel de Fellenberg n'envisageait d'accueillir ses six jeunes "Spartiates" dans son institution que pour mener avec eux une expérience à part, et déployait les plus grands efforts pour recruter à cet effet un maître aux compétences linguistiques et aux qualités de caractère si

19. Pour la lettre de Canaris, voir Chateaubriand, Mémoires d'outre-tombe, Paris, Le Livre de Poche, 1973 (1849-1850), 3 tomes, tome 2, p. 576-577. Pour celle de Tombazis, Bib. Genève, Ms. sup. 1885, fo $17-18$.

20. Le Constitutionnel, 4 et 7 mai 1826; Documents relatifs à l'état présent de la Grèce, nº 8, juilletnovembre 1827 , p. 52-53.

21. Bib. Genève, Ms. sup. 1891, fo 156-157.

22. Créée en 1798 par le quaker Joseph Lancaster et gérée par la British and Foreign School Society, c'était à la fois une école primaire et une école normale; on y pratiquait l'enseignement mutuel (voir Élie Halévy, Histoire du peuple anglais au XIXe siècle, 5 tomes, tome 1, L’Angleterre en 1815, Paris, Hachette, 1912, p. 504-505; Vernon Mallinson, "L'éducation en Grande-Bretagne et ses colonies», dans Gaston Mialaret et Jean Vial [dir.], Histoire mondiale de l'éducation, 4 tomes, tome 3, De 1815 à 1945, Paris, PUF, 1981, p. 165).

23. Réouvert en 1798 sur la Montagne Sainte-Geneviève par Victor de Lanneau, ce très ancien collège était un établissement privé, mais sous le contrôle de l'Université; les élèves suivaient les cours de Louis-le-Grand (voir Jules Quicherat, Histoire de Sainte-Barbe, collège, communauté, institution, Paris, Hachette, 1864, 3 tomes, tome 3).

24. La British and Foreign School Society comme Sainte-Barbe avaient une image libérale; Töpffer figure parmi les souscripteurs genevois (Bib. Genève, Ms. sup. 491, 8 décembre 1826). Sur sa pension, voir Thierry Groensteen, Benoît Peeters, Töpffer, l'invention de la bande dessinée, Paris, Hermann, 1994.

25. Pour Sainte-Barbe, Jules Quicherat mentionne seulement que «dans le grand monde libéral, on sollicita comme une faveur la permission de les faire sortir du collège les jours de congé» (Jules Quicherat, Histoire de Sainte-Barbe..., ouv. cité, p. 208-209). 
exceptionnelles que la mise en œuvre du projet en fut indéfiniment reportée ${ }^{26}$. L'autre modèle qui se dessine dans ce projet avorté - la création d'une institution spécifique - ne semble avoir inspiré qu'une réalisation : l'orphelinat de Bâle, dont nous savons peu de choses.

C'était pourtant la voie que préconisait pour y inscrire les plus hautes visées, Gian-Pietro Vieusseux, éditeur de la revue florentine L'Antologia : «[...] je regrette toutes ces éducations partielles, j’aurais voulu une entente générale pour former en Suisse, dans un endroit agreste, loin du luxe des villes et des salons, un institut semi-militaire [...] ${ }^{27}$. En déplorant des actions trop ponctuelles, Vieusseux posait le problème d'une stratégie éducative; le genre d'enseignement proposé visait à une stricte adéquation aux besoins présents de la Grèce; le lieu évoqué suggérait, plus qu'un décor ou un style, une ambition. La coopération technique mise en place par le réseau, dont j'ai parlé plus haut, ne devait pas être non plus l'«entente générale" voulue par Vieusseux ${ }^{28}$. Elle aurait supposé que les principaux comités donnent plus d'importance à cette forme d'action, ou des comités spécialisés ${ }^{29}$ de plus d'envergure. Pourtant, si aucune véritable stratégie n'a été adoptée par une instance capable de la mettre en œuvre, on a quand même débattu des besoins de la Grèce en la matière et donné parfois à ces "éducations partielles» des fondements idéologiques plus précis, des perspectives plus larges.

Avant tout, elle devait être utile. On s'accordait sur le mot; il prêtait à des interprétations différentes, d'autant que les établissements sollicités n'étaient pas de même niveau : école primaire (Lambeth) ou collège secondaire (Sainte-Barbe). En proposant d'instruire ces enfants «suivant leur état et leurs dispositions, dans les lettres et les sciences ou dans les métiers ${ }^{30}$, Guillaume Favre-Bertrand, du comité de Genève, se souciait, semble-t-il, plus du rang social et des aptitudes des élèves que des besoins de la Grèce ${ }^{31}$. Et sous ce rapport la balance n'était pas égale entre les deux orientations. La plupart ont reçu un enseignement «libéral $»^{32}$; les deux confiés à l'Institut agricole de Roville font figure d'exception; et on s'excusait sur une "prédilection bien marquée» de placer un élève de Lausanne en apprentissage

26. Bib. Genève, Ms. 3227, f $\mathrm{f}^{\circ}$ 69-70 et Ms. sup. 1885, fo 233-234.

27. Lettre à J.-G. Eynard, Bib. Genève, Ms. sup. 1885, fo 35-36.

28. Les comités n'ont jamais formé une véritable organisation, mais ont entretenu des correspondances régulières et coopéré sur des points particuliers. Dans ce cas-ci, il s'est agi surtout d'organiser l'acheminement des enfants à travers la France, la Suisse et l'Allemagne vers leur ville d'accueil.

29. La Société philhellénique du canton de Vaud, parallèle au comité grec de Lausanne, et la Société pour le réveil des mœurs et de la religion parmi les Grecs (Bâle), scission du comité de Bâle sur la base d'une perception plus religieuse que politique de la question (Bib. Genève, Ms. sup. 1885, fo 31-32).

30. Bib. Genève, Ms. 3228, f $\mathrm{f}^{\circ}$ 125-126.

31. Le choix correspondait à l'assise sociale du mouvement et s'imposait d'autant mieux qu'on pouvait voir dans ces enfants l'élite future du pays.

32. En France, très classiquement littéraire, les programmes n'ayant commencé à être modernisés qu'en 1829, (voir Maurice Gontard, L'enseignement secondaire..., ouv. cité, p. 153-156). 
chez un armurier ${ }^{33}$. Sentant le décalage avec la réalité grecque, à la Société vaudoise on répondait d'avance à ceux qui penseraient que «la Grèce réclame plutôt des artisans et des ouvriers que des hommes instruits [...] qu'il lui faut des uns et des autres; que la Grèce a des besoins matériels et des besoins moraux [...] "34. À Lambeth, plus concrètement, on entendait former des instituteurs pour la Grèce ${ }^{35}$; Fellenberg et Peschier l'auraient voulu aussi.

À cette idée d'utilité on associait donc, plus que le bénéfice personnel des enfants, l'intérêt du pays. L'un d'eux, selon le Journal des débats, devait recevoir "une éducation appropriée aux besoins de la Grèce, cet enfant se devant avant tout à sa patrie» ${ }^{36}$. L'adoption ne les en dispensait pas : l'architecte Mouchonnet promettait de faire du neveu de Canaris « un citoyen dont la Grèce [lui] aura[it] un jour l'obligation $»^{37}$. Et d'abord ils devaient connaître leur langue : l'orphelinat de Bâle se prévalait «d'un maître assez avancé dans la connaissance du Grec ancien pour lire avec eux les chefs-d'œuvre de cette langue et qui sera[it] mis à même de se rendre familière la langue moderne de la Grèce ${ }^{38}$. Fellenberg comptait aussi sur la chapelle grecque que le tsar entretenait chez lui pour les préserver «du malheur de devenir étrangers au culte de la religion, qui a, plus que tout autre moyen, contribué à leur conserver l'espérance de pouvoir reconquérir leur patrie»" ${ }^{39}$. Certain du reste "que le naturel distingué de la nation grecque a[vait] surtout besoin, que l'on isole ses générations naissantes vis-à-vis de Dieu et de la nature, au milieu du torrent de corruption qui les entraînent $[s i c] »^{40}$, Fellenberg, comme Vieusseux, ne voyait pas de moyen plus sûr de maintenir ces enfants dans leur langue et leur religion, de leur garder, dirions-nous, leur identité nationale, que de les tenir à l'écart de leur pays d'accueil.

C'était lier formation patriotique et éducation morale. Le modèle spartiate s'imposait là à l'évidence. En exil à Pise, le métropolite Ignace voulait, lui aussi, qu'on élève "les jeunes Grecs à la manière sobre des Spartiates" pour répondre aux nécessités d'un pays pauvre et ravagé par la guerre ${ }^{41}$. Mais l'impératif d'une éducation morale se suffisait à lui-même. "Société pour le réveil des mœurs et de la religion parmi les Grecs» : le nom est éloquent. Persuadé que «la liberté extérieure ne peut subsister sans la liberté intérieure»,

33. Bib. Genève, Ms. sup. 1891, fo 156-157.

34. Revue encyclopédique, tome 28, octobre 1825, p. 306.

35. Circulaire Éducation en Grèce. Appel fait au Public par la Société des Écoles pour la Grande Bretagne et l'Étranger, au sujet de l'Éducation en Grèce, Bib. Genève, Ms. sup. 1891, fo 164.

36. Le Journal des débats, 7 mai 1826. C'est moi qui souligne.

37. Documents relatifs à l'état présent de la Grèce, no ${ }^{8}$, juillet-novembre 1827 , p. 53. Pourtant certains orphelins recueillis aux États-Unis sont devenus citoyens américains; l'un d'eux fut même représentant du Wisconsin (Stamati Théodore Lascaris, "Le philhellénisme américain, 1821-1828», L'Acropole, $\mathrm{n}^{\circ} 17-18$, janvier-juin 1930, p. 108).

38. Bib. Genève, Ms. 3228, fo 18-19.

39. Bib. Genève, Ms. 3227, fo 69-70.

40. Bib. Genève, Ms. 3227, f 69 -70.

41. Lettre à J.-G. Eynard, Bib. Genève, Ms. sup. 1888, fo 226-227. 
on y avait "principalement en vue l'état moral et religieux de la nation $»^{42}$. Invoquant aussi la religion, non plus comme ferment d'identité nationale, mais pour un fonds commun de moralité prêté au christianisme, toutes dénominations confondues, la British and Foreign School Society, où se mêlaient quakers, évangélistes et disciples de Bentham, préconisait l'instruction religieuse comme moyen de «faire germer l'esprit des vertus privées et des vertus publiques». Par l'influence que les jeunes gens, devenus des «instruments de culture et de civilisation $»^{43}$, exerceraient sur leurs compatriotes, on pensait "accélér[er] la régénération morale des Grecs» ${ }^{44}$. Liant plus intimement encore régénération morale et édification politique, Fellenberg disait vouloir "préparer une bonne éducation nationale aux peuples de la Grèce $»^{45}$. Autrement dit, former ici, en Suisse, comme en laboratoire, une petite élite qui pourrait ensuite, là-bas, en Grèce, plus que transmettre un savoir, servir d'exemple. D'où l'exigence de concentrer son action sur un petit groupe d'enfants très jeunes avec qui il pourrait suivre «une marche soigneusement graduée, et combinée avec beaucoup de soins, dans tous ses rapports» ${ }^{46}$. Une marche lente et discrète, toute pédagogique, avec pourtant une ample visée : aux antipodes des initiatives du comité de Paris, qui se bornait à faire bénéficier des enfants aux noms prestigieux d'une éducation toute classique, mais avec la plus grande «ostentation $»^{47}$.

\section{LES RAISONS D'UNE PRÉDILECTION}

Si on se demande à présent pourquoi de telles initiatives ont paru particulièrement appropriées pour soutenir cette cause, on pensera d'abord à la conjonction d'une demande et d'une offre : l'inclination de certains philhellènes pour la pédagogie rencontrait un fort appétit d'instruction chez les Grecs. De celui-ci témoignait la présence d'étudiants grecs dans les universités européennes, ancienne, mais plus massive depuis le siècle précédent. Le plus illustre d'entre eux, Adamantios Koraïs, qui y voyait l'effet de l'enrichissement par le commerce maritime, avait attiré, vingt ans plus tôt, l'attention sur cette pratique ${ }^{48}$. Le comité de Paris a pu s'en inspirer, et certains notables n'ont cherché qu'à lui assurer la protection du mouvement philhellène en

42. Bib. Genève, Ms. 3228, f $\mathrm{f}^{\circ}$ 16-17 et $18-19$. Voir aussi note 34.

43. Bib. Genève, Ms. 3228, fo 18-19.

44. Circulaire Education en Grèce..., Bib. Genève, Ms. sup. 1891, fo 164.

45. Bib. Genève, Ms. sup. 1885, fo $233-234$.

46. Bib. Genève, Ms. sup. 1885, fo 252-253.

47. Bib. Genève, Ms. sup. 1886, fo $1-2$.

48. Adamantios Koraïs, Mémoire sur l'état actuel de la civilisation dans la Grèce, lu à la Société des Observateurs de l'homme le 16 nivôse an XI, s.l.n.d. Ce phénomène participait aussi des "Lumières grecques", sur lesquelles on consultera Astérios Argyriou, "Au "siècle des Lumières grecques" : renouveau intellectuel et conscience orthodoxe dans les Balkans", dans Jean-Marie Mayeur, Charles et Luce Piétri, André Vauchez, Marc Venard [dir.], Histoire du christianisme, tome 10, Les défis de la modernité (17501840), Paris, Desclée, 1997, p. 146-150. 
recommandant leurs enfants aux comités ou à des personnalités sympathisantes. Parce qu'elle mettait en avant un intérêt collectif, une démarche du bey du Magne, Pierre Mavromichalis, semble avoir été plus proche des vues pédagogiques des philhellènes : jugeant que "c'[était] faire tort à l'humanité que de ne pas cultiver l'esprit plein de vivacité des enfants spartiates», il souhaitait en voir une quinzaine pris en charge par les comités suisses ${ }^{49}$. Pourtant, le projet qu'on avait eu d'abord à Genève de s'orienter dans cette direction avait reçu un accueil réticent : les envoyés grecs à Londres, tout en saluant l'idée généreuse, lui avaient opposé que la situation présente (les troupes égyptiennes ravageaient la Morée, assiégeaient Missolonghi) réclamait des "secours plus immédiates [sic] et plus utiles " ${ }^{50}$. Autrement dit, comme l'enfant du poème de Hugo, ils voulaient «de la poudre et des balles " ${ }^{51}$. C'était donc aussi la réaction des Grecs qui avait fait cantonner ces projets dans une position subalterne.

Du côté des philhellènes, on attribuerait volontiers l'ardeur pédagogique à la qualité de certains : Emmanuel de Fellenberg, Adolphe Peschier, futur professeur à Tübingen, les enseignants qui formaient la Société philhellénique du canton de Vaud, une institution spécialisée comme la British and Foreign School Society. Pourtant, la place des enseignants dans le mouvement n'a pas été si considérable. Aucune commune mesure entre leur contribution à la souscription du comité de Paris et celle des gens de loi ou du monde de la finance, de l'industrie et du commerce; et le rôle des écoles et des universités dans le mouvement a été mineur au regard de celui des salons parisiens, des Églises protestantes ou des loges maçonniques. En fait, cette propension pédagogique, loin d'être portée par les seuls milieux académiques, exprimait une préoccupation qui travaillait plus globalement les sociétés européennes.

Il faut donc rapporter ces initiatives à l'intérêt général pour l'éducation, tel qu'il se manifestait alors. Notons d'abord que le cadre scolaire semble être allé de soi; on aurait pu pourtant imaginer, pour ces quelques enfants de notables, de faire appel à des précepteurs. Selon une distinction familière aux débats de la Révolution française, le modèle démocratique de «l'éducation commune» l'avait emporté sur celui, aristocratique, de «l'éducation domestique». Néanmoins, l'idée de Pestalozzi, que «les avantages présentés par l'éducation domestique devaient être imités par l'éducation publique " ${ }^{52}$, avait laissé des traces : l'ambiance familiale à Lausanne, le petit nombre d'élèves voulu par Fellenberg. Pour celui-ci, c'était l'effet de la volonté nette d'inscrire son programme d'instruction dans un projet plus global d'éduca-

49. Bib. Genève, Ms. sup. 1884, fo 260-261.

50. Bib. Genève, Ms. 3227, fo 9-10.

51. "L'Enfant», dans Les Orientales, Paris, Charles Gosselin, 1829, p. 187-192.

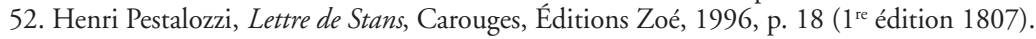


tion - autre distinction chère aux révolutionnaires français ${ }^{53}$. D'ailleurs, pour ceux qui réfléchissaient alors à ces questions - Jeremy Bentham et ses amis ou Marc-Antoine Jullien, directeur de la Revue encyclopédique, qui faisait figure d'expert sur le sujet ${ }^{54}$-, éducation et instruction concourraient au même but : l'utilité comme convergence de l'intérêt public et du bonheur privé. Ce qu'on visait aussi pour ces enfants grecs. On s'accordait moins sur les moyens d'y parvenir. Croyant avec Helvétius à l'égalité des aptitudes naturelles et à la toute-puissance de l'éducation, les benthamiens voulaient un modèle d'éducation unique; Jullien pensait qu'on pouvait seulement développer des aptitudes irréductibles, et pour chacun différentes. Alors, pour lui, l'harmonie des biens privés et publics passait par la division du travail et un système éducatif différencié, dont Hofwil offrait le modèle avec un collège pour l'élite, une école pour les travailleurs de l'industrie, une autre pour les agriculteurs. Eût-il été réalisé, le projet «spartiate» n’aurait fait qu'y ajouter une spécialisation. Mais, comme Peschier ou comme les disciples de Lancaster, il entendait aussi former des formateurs, s'inscrivait donc dans une perspective de généralisation de l'instruction. Rien pourtant de plus contraire à sa «marche soigneusement graduée» que le développement rapide de l'instruction élémentaire dans les classes populaires visé à Lambeth, et le style d'application massive, la délégation de l'enseignement à des moniteurs qu'on y pratiquait ne s'opposaient pas moins à la haute idée que, fidèle à l'esprit de Pestalozzi, Fellenberg se faisait du rôle personnel du maittre, au point, on l'a vu, de faire de son "caractère" une condition indispensable de son projet.

En France, le choix du cadre scolaire impliquait de s'inscrire dans le système d'instruction publique d'État : comme leurs condisciples de SainteBarbe ou des pensions où ils étaient reçus, les jeunes Grecs ont dû suivre les cours des collèges royaux. Ailleurs, on a eu recours à des établissements privés. Mais les vues de la British and Foreign Society, de la Société bâloise ou de Fellenberg ne démentaient pas l'évolution qui avait fait, selon Marcel Gauchet, de l'éducation « une préoccupation civique et une affaire publique de premier plan ${ }^{55}$. L'initiative pouvait être privée, l'enjeu ne l'était pas. Si Marc-Antoine Jullien, se passionnant pour les questions pédagogiques dans les années 1800, s’intéressait aux expériences des pédagogues suisses, non aux lycées napoléoniens, sa démarche restait foncièrement politique : il cherchait un palliatif à ce qu'il pensait avoir été l'échec de la Révolution française. Il renouait aussi avec la tradition de la philanthropie qui avait fait

53. Voir l'introduction de Bronislaw Baczko au recueil Une éducation pour la démocratie. Textes et projets de l'époque révolutionnaire, Genève, Droz, 2000.

54. Il a publié entre 1808 et 1817 cinq ouvrages sur le sujet, dont un Essai sur l'emploi du temps souvent réédité et deux à propos de Pestalozzi et Fellenberg. Voir le livre de Robert Roswell Palmer, From Jacobin to Liberal. Marc-Antoine Jullien, 1775-1848, Princeton, Princeton University Press, 1993.

55. "Démocratie, éducation, philosophie», dans Marie-Claude Blais, Marcel Gauchet, Dominique Ottavi, Pour une philosophie politique, Paris, Bayard, 2002, p. 30. 
de l'éducation populaire un de ses domaines d'intervention ${ }^{56}$, et se définissait plus généralement comme la prise en charge par des particuliers de certains objets d'intérêt public. Et le mouvement philhellène dans son ensemble relevait de cette tradition ${ }^{57}$; seulement, le décalage y était plus marqué entre l'initiative privée et un objet par excellence de nature politique : l'indépendance d'un peuple, la construction d'une nation. Un caractère hybride qu'exprimait bien l'ambition de Fellenberg : "préparer une bonne éducation nationale aux peuples de la Grèce» en éduquant six enfants grecs. Souvent associées, on l'a vu, à la compassion pour les victimes, ces initiatives faisaient toutefois jouer davantage le ressort philanthropique du philhellénisme. Il est significatif à cet égard qu'elles se soient manifestées tantôt dans les débuts de certains comités, quand ils ne se sentaient pas assez sûrs d'être soutenus pour envisager une action plus ouvertement politique, tantôt sur les retombées de la mobilisation, quand, au comité de Londres, on ne croyait plus dans une victoire des Grecs, ou quand, à l'inverse, leur indépendance a paru acquise.

L'affinité entre philhellénisme et préoccupation éducative était pourtant bien plus intime que ne le ferait croire cette position marginale. La culture antique d'abord avait une telle place dans l'enseignement qu'un lien ne pouvait manquer de se créer dans les esprits entre la "terre classique» et l'école ${ }^{58}$. Mais l'attache était encore plus précise, plus centrale, juste au croisement de deux traits majeurs de l'idéologie philhellène : l'idée d'une dette de l'Europe envers la Grèce, la conception des événements grecs comme une régénération.

Le motif de la dette envers la Grèce antique a été un des plus constants lieux communs du discours philhellène. Dès l'automne 1821, dans un des premiers appels à souscription, Ambroise Firmin-Didot, futur membre du comité de Paris, évoquait les Grecs exilés après la chute de Constantinople, qui "donnèrent à l'Italie, en échange de l'hospitalité, les connaissances précieuses dont ils étaient demeurés les dépositaires» et concluait : «L'Europe, en acceptant le bienfait, a contracté envers les Grecs une dette sacrée : le moment est venu de l'acquitter " $"$. Le mot, avec sa charge d'obligation, fut repris inlassablement pour justifier toute forme de soutien. Mais le plus

56. Voir l'action du pasteur Oberlin au Ban de La Roche ou les initiatives de La Rochefoucauld à Liancourt (Catherine Duprat, Pour l'amour de l'humanité. Le Temps des philanthropes. La philanthropie parisienne des Lumières à la monarchie de Juillet, Paris, Comité des travaux historiques et scientifiques, 1993, p. 33-36).

57. Comme le soulignait le nom de l'association, dont le Comité grec de Paris n'était en principe que le comité central : Société philanthropique en faveur des Grecs.

58. La révolte des Grecs n’aurait pas rencontré un tel écho sans cette présence vive entretenue aussi dans les esprits par un goût néo-classique né autour de 1770 et que le romantisme naissant - en France n'avait pas encore supplanté, les deux styles coexistant de façon frappante dans la poésie et la peinture philhellènes. La fixation d'un Fellenberg sur les jeunes Spartiates renvoyait aussi à l'imaginaire politique de la Révolution française à laquelle il avait été mêlé. [idot].

59. Souscription française en faveur des Grecs, Paris, s.d. [1821]. Texte signé A.[mbroise] F.[irmin]-D. 
approprié n'était-il pas de rendre à leurs descendants le trésor de savoir hérité de leurs ancêtres? Comme beaucoup, Fellenberg en était persuadé : "[...] nous devons tâcher d'acquitter la dette, que nous a imposée la Grèce antique, en fournissant à la Grèce moderne les éléments les plus essentiels d'une restauration morale complète, et enrichie par ce que nos progrès dans les sciences et les arts ont de bienfaisant ${ }^{60}$. Soulignons la formule finale : on ne devait pas seulement rendre aux Grecs ce qui avait été reçu de leurs ancêtres; il fallait aussi les faire profiter, comme un intérêt de la dette, de ce que l'Europe avait ajouté à l'héritage. Et on précisait à Lausanne qu'on souhaitait offrir en exemple à ces enfants «[...] nos institutions civiles et militaires, notre éducation publique, nos études, notre vie, [...] celles d'un peuple libre comme les Grecs veulent l'être, républicain comme l'étaient leurs aïeux $»^{61}$.

Rendre aux Grecs ce qu'on avait reçu de leurs ancêtres paraissait d'autant plus opportun qu'on les voyait, en se soulevant, renouer d'eux-mêmes avec cet héritage. Dans l'aide éducative, la solidarité fondée sur l'idée d'une dette à acquitter rejoignait la pensée que le combat des Grecs valait régénération. Mais l'articulation des deux thèmes en faisait jouer aussi les ambivalences. L'appel de la British and Foreign School Society, déjà cité, le montre bien, qui commençait par évoquer «ce pays jadis le plus célèbre dans les annales de la civilisation [...] de nouveau réveillé du sein de la dégradation pour s'élancer, plein de joie et d'espérance, vers la conquête de ses libertés", et s'achevait sur l'espoir de voir "la régénération morale de la Grèce nous offrir bientôt [...] la récompense la plus flatteuse et la plus douce de nos travaux " $"{ }^{62}$. L'image du réveil évoquait d'abord un événement ponctuel, déjà survenu, le retour acquis de la Grèce à son glorieux passé; et puis, l'accent se déplaçait vers le futur, vers quelque chose qui restait à espérer et n'adviendrait que comme le prix d'efforts à accomplir : la régénération. D'un côté l'élan; de l'autre le travail. La régénération comme grâce et la régénération comme tâche, les deux valeurs entre lesquelles a oscillé, selon Mona Ozouf, l'idée que ses acteurs se faisaient de la Révolution française ${ }^{63}$. De même, voyant dans l'émergence des héros de l'indépendance dans une Grèce si peu instruite une sorte de miracle, le métropolite Ignace pensait que les Grecs devaient désormais travailler à «donner à la génération future une éducation religieuse et morale, [pour] les mettre à même de jouir de la liberté [...] " ${ }^{64}$. Prolongement indispensable de l'élan premier, nécessaire, disait encore Fellenberg, "pour la consolida-

60. Bib. Genève, Ms. 3227, fo 69-70.

61. Revue encyclopédique, tome 28, octobre 1825, p. 307.

62. Bib. Genève, Ms. 3227, fo 69-70.

63. Mona Ozouf, «La Révolution française et la formation de l'homme nouveau », dans L'homme régénéré. Essais sur la Révolution française, Bibliothèque des histoires, Paris, Gallimard, 1989, p. 116157 et «Régénération» dans François Furet et Mona Ozouf [dir.], Dictionnaire critique de la Révolution française, Paris, Flammarion, 1988, p. 731-741.

64. Bib. Genève, Ms. sup. 1888, fo 226-227. 
tion de ses destinées futures ${ }^{65}$, l'entreprise éducative relevait clairement de la régénération entendue comme un travail. Annonçant, vingt ans plus tôt, à l'Europe une "régénération commencée» de la Grèce, Koraïs avait vu le développement de l'instruction comme un long effort, qui devait être poursuivi, puisque n'étaient "pas encore arrivés pour [la Grèce] ces temps heureux où [elle serait] l'émule de l'ancienne Grèce „ ${ }^{66}$. Et il voyait une éventuelle révolution politique comme l'aboutissement logique de cette régénération intellectuelle et morale ${ }^{67}$. À l'inverse, c'était parce qu'il ne croyait plus aux chances de la révolution politique, que John Bowring, du comité de Londres, conseillait au nouveau comité de Genève de réserver ses efforts à l'éducation de jeunes Grecs, comme le seul moyen de créer «un futur plus fortuné pour la Grèce " ${ }^{68}$.

Quels qu'aient été ses rapports avec la révolution politique, le souci de l'éducation faisait pencher le motif de la régénération du côté du travail. Mais l'ambiguiité ressurgit, irréductible cette fois, si on regarde à qui la tâche incombait, vers quoi tendait l'effort. Dans l'appel de la British and Foreign School Society le contraste est net entre, ici, un mouvement spontané des Grecs et, là, un travail d'éducation qui était celui de l'association anglaise. Usant de la même image du réveil, Koraïs décrivait une révolution éducative dont les Grecs ne devaient qu'à eux-mêmes et l'initiative et l'effort, tandis que John Bowring semblait reporter tous ses espoirs sur une action à long terme des philhellènes. Il est vrai que sa désillusion, comme chez beaucoup, portait précisément sur la capacité des Grecs à mener à bien leur révolution. La domination ottomane avait maintenu le gros de la population grecque dans l'ignorance, elle l'avait surtout corrompu moralement. Aussi la situation exigeait-elle en plus d'"une instruction sagement répandue", "un système d'éducation capable d'extirper les causes, et d'effacer les effets d'une domination dévastatrice " ${ }^{69}$. De ceux-ci, les pires étaient les atrocités commises par certains Grecs, si semblables à celles qui symbolisaient aux yeux de l'Europe la barbarie turque. S'en indignant, Maxime Raybaud, un des volontaires français venus combattre à leurs côtés, trouvait un motif d'espérer que «plus éclairés, [les Grecs] deviendr[aie]nt plus

65. Bib. Genève, Ms. sup. 1885, fo 221.

66. Adamantios Koraïs, Mémoire sur l'état actuel..., ouv. cité, p. 64.

67. Stephen G. Chaconas voit un véritable "plan pour l'indépendance de la Grèce» à l'œuvre dans sa publication des classiques (Adamantios Koraïs, a Study in Greek Nationalism, New York, Columbia University Press, 1942, p. 35). Le lien avec la révolution politique reste néanmoins problématique chez Koraïs, et Dimitri Nicolaïdis a pu écrire que «sa vision du progrès semble se réduire à un développement sans bornes "des sciences et des arts", mouvement autonome où la politique n'intervient que négativement, comme contrainte, au mieux comme moyen" (D'une Grèce à l'autre. Représentation des Grecs modernes par la France révolutionnaire, Paris, Les Belles Lettres, 1992, p. 230-231).

68. Bib. Genève, Ms. 3228, fo 84-86.

69. Circulaire Éducation en Grèce... Ce prosélytisme, ici ou à Bâle, annonçait la campagne missionnaire des Églises réformées après l'indépendance, qui ferait hésiter l'Église grecque entre raidissement identitaire et ouverture relative (voir Astérios Argyriou, «Église orthodoxe et insurrections nationales dans les Balkans", dans Jean-Marie Mayeur et al. [dir.], Histoire du christianisme..., ouv. cité, p. 759768). 
humains» dans l'affliction où ces actes plongeaient ceux des Grecs qui avaient, écrivait-il, "habité nos villes et pratiqué nos usages»" ${ }^{70}$. Comme si la régénération de la Grèce n'avait eu d'autre aboutissement, en fin de compte, que l'assimilation des Grecs aux Européens. Une ambiguïté qui n'affectait pas moins Koraïs, qu'on tient pour l'inspirateur d'un "nationalisme grec» avant la lettre ${ }^{71}$. Ses efforts pour rendre aux Grecs une langue épurée, authentiquement la leur, le rapprochaient de Herder; mais il tenait pour régénérateurs le séjour en Europe d'une partie de la jeunesse ou la multiplication des traductions en grec d'ouvrages étrangers. Il appelait ses compatriotes à «rentrer en possession des lumières de [leurs] ancêtres $»^{72}$, y contribuait par sa grande édition du corpus classique, mais pour qu'ils reprennent leur place dans l'Europe éclairée, qu'ils participent à un progrès des Lumières ou de la civilisation dont l'horizon ne pouvait être qu'universel.

\section{Des PROLONGEMENTS SIGNIFICATIFS SUR LE TERRAIN EN GRÈCE}

En regard d'un enracinement si profond dans la configuration idéologique du philhellénisme, l'éducation en Europe de quelques dizaines d'enfants paraît un mince accomplissement. L'écart semblera moins grand si on considère les manifestations de cette visée éducative dans l'intervention des philhellènes en Grèce même. J'en donnerai trois exemples.

Inquiets du «triste état des enfants qui au milieu de cette lutte prolongée grandissent sans surveillance et sans éducation ", les responsables de la Société bâloise auraient voulu «travailler sur les lieux mêmes à l'éducation morale et religieuse de cette jeunesse abandonnée " ${ }^{73}$. Les "obstacles» leur avaient paru trop grands dans l'immédiat, mais la British and Foreign School Society avait pu annoncer, comme elle l'avait prévu, le retour en Morée d'un des enfants élevés à Lambeth "accompagné d'un gentilhomme, que des motifs de pure bienfaisance [avaient] engagé à s'expatrier pour aller au loin se consacrer aux intérêts de l'éducation $»^{74}$; ils avaient pour mission "l'établissement d'une école fondée d'après le système anglais, au siège du gouvernement grec». Nous savons peu de choses de l'action scolaire d'Edward Masson - le «gentilhomme» en question; nous ne savons pas non plus s'il y a eu une école d'enseignement mutuel à Nauplie. Mais à Athènes en 1826, un jeune philhellène français, François-Blaise Schack, en a vu fonctionner deux, «l'une pour le sexe, l'autre pour les jeunes garçons", et à une assez grande échelle, puisque

70. Maxime Raybaud, Mémoires sur la Grèce pour servir à l'histoire de la guerre de l'indépendance, Paris, 1824-1825, 2 tomes, tome 1, p. 513-514.

71. C'est la thèse de Stephen G. Chaconas.

72. Adamantios Koraïs, Mémoire sur l'état actuel..., ouv. cité, p. [I].

73. Bib. Genève, Ms. 3228, fo 18-19.

74. Circulaire Éducation en Grèce, ouv. cité. 
l'école des garçons aurait compté plus de deux cents élèves. La méthode était anglaise, les maîtres grecs, et Schack y a vu distribuer des livres scolaires envoyés par le comité de Paris ${ }^{75}$. Plus tard, en 1829, "un des premiers soins » du médecin américain Samuel Howe, quand il fonda une colonie agricole à Hexamilia, près de Corinthe, fut d'y ouvrir une école, confiée à un jeune Grec qui avait appris la méthode de Lancaster en Angleterre : «les visages brillants, l'attention avide et l'étonnante sagacité des petits gamins, [...] indications certaines du caractère futur de la race» le consolaient, disait-il, de toutes les contrariétés ${ }^{76}$.

Pour Leicester Stanhope, qui a représenté le comité de Londres en Grèce pendant le premier semestre de 1824, "trois ou quatre" maîtres d'école auraient pu répandre "le système de Lancastre avec autant de rapidité que se succèdent les conquêtes de la Grèce $»^{77}$. Mais le programme éducatif de ce colonel britannique était autrement plus vaste. Ainsi il avait fixé pour but à la Société Philo-Muse, fondée par lui à Athènes, d' «établir des écoles, des musées, des pharmacies, des sociétés d'agriculture, et enfin tout ce qui peut contribuer à répandre les connaissances $»^{78}$. Il tenait particulièrement à la création d'une poste "qui donnerait cours aux idées des hommes»" ${ }^{79}$. Du reste, il comprenait sa mission comme l'occasion d'expérimenter les théories de Bentham sur cette terre vierge que la Grèce lui semblait offrir ${ }^{80}$. Toutefois, malgré l'appui d'Odysseus Androutsos, alors maître de l'Attique, pour qui il s'était pris d'enthousiasme, il n'a guère fait que distribuer un peu partout des presses lithographiques pour la création de journaux, méritant par là le sobriquet de «typographical colonel» dont la verve ironique de Byron l'avait affublé.

Plus que dans les vues de ce personnage ou dans de rares réalisations scolaires, on trouvera la manifestation significative de la volonté éducative des philhellènes dans leur effort, soutenu, pour former une armée régulière. Ce point-là était tout à fait central : la première urgence pour la Grèce était d'achever de conquérir son indépendance; les «comités grecs» voyaient dans la formation d'une armée à l'européenne le seul moyen d'y parvenir; la plupart des volontaires étaient venus d'abord pour combattre. Et c'était aussi affaire d'éducation : on devait inculquer le modèle européen - la discipline, le combat en ligne - à des hommes habitués à la guérilla et organisés en bandes, aux effectifs fluctuants, regroupées autour d'un capitaine.

75. F.[rançois]-R. Schack, Campagne d'un jeune Français en Grèce, envoyé par M. le duc de Choiseul, Paris, Didot, 1827, p. 88-89.

76. Samuel Gridley Howe, Letters and Journals During the Greek Revolution, London, J. Lane, Boston, D. Estes, 1907-1909, 2 tomes, tome 1, p. 356.

77. Lettres du colonel Stanhope sur la Grèce, Paris, Corneille, 1825, p. 130.

78. Idem, p. 170.

79. Idem, p. 68-69.

80. Frederick Rosen conteste la légitimité de sa référence à la pensée de Bentham dans Bentham, Byron and Greece: Constitutionalism, Nationalism and Early Liberal Political Thought, Oxford, Clarendon Press, 1992. 
Ce même dessein se retrouve dans les initiatives militaires les plus diverses des philhellènes. En organisant à Missolonghi un corps d'artillerie, Byron ne visait pas tant à doter ses membres d'un savoir-faire qu'à les «accoutumer à la discipline ${ }^{81}$; le comité de Paris pensait y contribuer par l'envoi en Grèce d'uniformes et d'instruments de musique ${ }^{82}$. Formé en corps séparé, le «bataillon des Philhellènes», qui regroupa un temps la plupart des volontaires européens avant d'être décimé lors du désastre de Péta ${ }^{83}$, aurait dû aussi "servir d'école militaire pratique à de jeunes Grecs ${ }^{84}$. L'expérience la plus conséquente a été celle du corps des Tacticoi, que le colonel Fabvier avait créé à l'été 1825, à la demande du gouvernement grec, comme un embryon d'armée régulière, qu'il a dirigé trois ans, et qui a compté jusqu'à 3700 hommes ${ }^{85}$. En plus du rôle d'instructeur dévolu aux volontaires européens - en dépit de leur méconnaissance de la langue -, deux traits font ressortir la dimension éducative de l'expérience. Pour soustraire ses hommes à l'atmosphère délétère de Nauplie, Fabvier les a installés dans un lieu isolé, la presqu'île de Methana, rebaptisée Tacticopolis - un peu comme Fellenberg l'avait imaginé pour ses jeunes Spartiates, ou comme, trente ans plus tôt, la Convention avait établi son éphémère école militaire, l'École de Mars, dans la plaine des Sablons, à l'écart des "vices d'une grande ville " ${ }^{86}$. Un ancien des armées napoléoniennes, Adolphe Perrot, qui avait fait partie des Tacticoi, a décrit aussi «les peines et les efforts de Fabvier pour [...] convaincre [ses hommes] que sans obéissance il ne peut y avoir d'ordre parfait " ${ }^{87}$, en des termes qui évoquent autant que l'autorité personnelle du chef militaire, celle du maître pédagogue.

Ces efforts de Fabvier, Perrot les admirait, mais doutait de leur réussite. D'autres ont remis en cause le bien fondé de l'entreprise, jugeant vain de vouloir imposer aux Grecs le modèle européen, celui qu'ils suivaient étant mieux adapté à leur tempérament et à leur pays. On pourrait opposer à la figure de Fabvier, celle de l'Américain George Jarvis, qui a mené plusieurs années la vie d'un «capitaine». Au reproche d'être «trop grec», il opposait son désir «de passer pour l'un d'eux et d'acquérir toutes leurs bonnes qualités ${ }^{88}$. Exceptionnel, pareil renversement est pourtant révélateur de la position incertaine de philhellènes, qui, au rebours de leurs attentes, trouvaient chez

81. Pietro Gamba, Relation de l'expédition de Lord Byron en Grèce, Paris, Peytieux, 1825, p. 186.

82. F[rançois]-R. Schack, Campagne d'un jeune français..., ouv. cité, p. 44.

83. Le 16 juillet (4 juillet dans le calendrier grec) 1822, au cours d'une expédition pour étendre l'insurrection en Épire.

84. Maxime Raybaud, Mémoires..., ouv. cité, tome 2, p. 240.

85. Sur le corps des Tacticoi et en général sur l'aspect militaire du philhellénisme, voir Antonin Debidour (Le Général Fabvier, sa vie militaire et politique, Paris, Plon, 1904), Douglas Dakin (British and American Philhellenes during the War of Greek Independence 1821-1833, Thessalonique, Institut for Balkan Studies, 1955), William Saint Clair, et le chapitre 9 de mon livre.

86. Cité par Bronislaw Baczko, Une éducation pour la démocratie, ouv. cité, p. 40.

87. Adolphe Perrot, Relation d'un voyage en Grèce dans les années 1826 et 1827, Bordeaux, impr. R. Laguillotière, 1827 , p. 15.

88. Lettre datée de mai 1824 publiée par William Parry dans The Last Days of Lord Byron, Paris, Gali- 
les Grecs modernes autant que l'héritage de la Grèce ancienne l'influence de leurs maitres ottomans. Révélateur aussi de ce que le projet philhellène avait de profondément paradoxal : aider les Grecs à devenir (ou redevenir) euxmêmes, par eux-mêmes - c'est-à-dire à rejoindre, comme l'une d'entre elles, le concert des nations européennes.

Denys Barau est docteur en études politiques (EHESS) et ancien attaché de conservation aux Archives départementales de la Loire

gnani, 1826, reproduite dans George Jarvis, Journal and related documents, édition établie par George G. Arnakis, Thessaloniki, Institute for Balkan Studies, 1965, p. 233. 